\title{
Speed Sign Recognition using Shape-based Features
}

\author{
Jafar Abukhait \\ Tafila Technical University \\ P. O. Box 179 \\ Tafila 66110, Jordan
}

\author{
Imad Zyout \\ Tafila Technical University \\ P. O. Box 179 \\ Tafila 66110, Jordan
}

\author{
Ayman M Mansour \\ Tafila Technical University \\ P. O. Box 179 \\ Tafila 66110, Jordan
}

\begin{abstract}
An efficient shape-based recognition system of U.S. speed limit road signs is presented in this paper. The proposed system accomplishes speed sign detection and recognition processes using three main stages, namely, geometrical-based detection of rectangular road signs, shape-based segmentation and feature extraction, and pattern classification using a K-nearest neighbor classifier (KNN). Twenty shape descriptors are computed for the most discriminative numerals of each detected sign. The proposed system is invariant to scale, rotation, and partial occlusion. The proposed system has been tested in different conditions, including sunny, cloudy, and rainy weather, and the experimental results on 195 speed signs reveals the efficiency of the proposed shape pattern segmentation and feature extraction methods.
\end{abstract}

\section{General Terms}

Speed Sign Recognition, Morphology-based Features, Feature Extraction, Classification.

\section{INTRODUCTION}

Driver assistant systems (DAS) and autonomous vehicles have been suggested by transportation departments due to high investment in road network management. The goal of such systems is to make driving safer and more reliable [1]. Such systems can benefit from road signs information by applying artificial intelligence and computer vision techniques.

Nowadays, automated road sign detection and recognition systems are being proposed since road signs have a significant role in regulating traffic and warning drivers over highways. Detection and recognition are considered as two different and cascaded problems. The purpose of detection is to maintain sign objects and remove non-sign objects from road sign frames while recognition is used to classify different road signs to their categories.

In general, detection of road signs is accomplished using: colorbased, shape-based, or integrated methods that use both color and shape information simultaneously. Sign's color information is used in color-based methods to remove non-road sign objects from the scene. While color thresholding in RGB is being used to segment road sign images ([2], [3], [4]), other researchers use Hue Saturation Intensity (HSI) space in the segmentation process ([5], [6]).

Shape-based detection methods are being deployed to detect road signs objects including speed sign ones. In [7, 8], distance to border (DtB) vector is used to build the shape feature vector which is used in shape classification by SVM, while principal component analysis (PCA) and k-nearest neighbor (KNN) classifier are used to detect the sign in [4]. Hough transform and radial symmetry are used to recognize triangular and circular shape road signs in [9]. Haar-like features are used in [10] while Genetic algorithm is used in [5] to detect road sign shape. In [13], fast radial symmetry is used to detect circular speed signs from a gray-scale image without using color information. In [14], a self-organizing map (SOM) is used on Gabor wavelet convoluted images to distinguish road signs from non-road signs.

In the classification, different techniques were suggested to improve classification accuracy and processing time. Many road sign recognition systems use Artificial Neural Network (ANN) in the classification process ([5], [6], [15], [16], [17]). Template matching using cross correlation is also used to identify road sign objects ([4], [8], [18], [19]).

Statistical, structural, or spectral features along with different classifiers are used in road sign recognition. Histogram is used as an image descriptor in [20] while Principal Component Analysis (PCA) is used in [4]. Zernike moment [21], Scaleinvariant feature transform (SIFT) [22], and color distance transform (CDT) ([23], [24]) are also deployed as image descriptors. Support vector machine (SVM) ([2], [21]), Knearest neighbor (KNN) [4, 20, 22], Forest Error-Correcting Output Code (F-ECOC) [9], and Bayesian generative modeling [25] are used as classifiers in the road sign recognition process.

This work is intended to implement an automated system for the recognition of speed limit signs in the United States. The proposed system has the ability to classify six different categories of rectangular shape speed signs: Speed Limit 15 , Speed Limit 25, Speed Limit 35, Speed Limit 45, Speed Limit 55 , and others (which include all rectangular white signs other than the previous five speed signs) as shown in Figure 1. The proposed system, automatically, segments the numerals of the speed sign using morphological operations; then different shape-based features of these numerals are extracted to build a feature vector that is used by a $\mathrm{KNN}$ classifier.

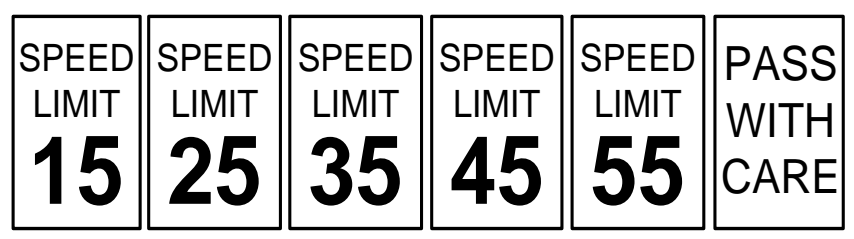

Figure 1: The six sign categories tested by our proposed system.

\section{PROPOSED SHAPE-BASED SIGN RECOGNITION SYSTEM}

The proposed automated detection and recognition system of U.S. speed signs, as shown in Figure 2, follows five stages.

1. Color-based segmentation: speed sign objects are extracted by converting sign images to achromatic ones and applying RGB color thresholding.

2. Rectangular speed sign shape detection: this stage discard non-speed sign objects and detects rectangular shapes using a set of cascaded geometric detectors. 


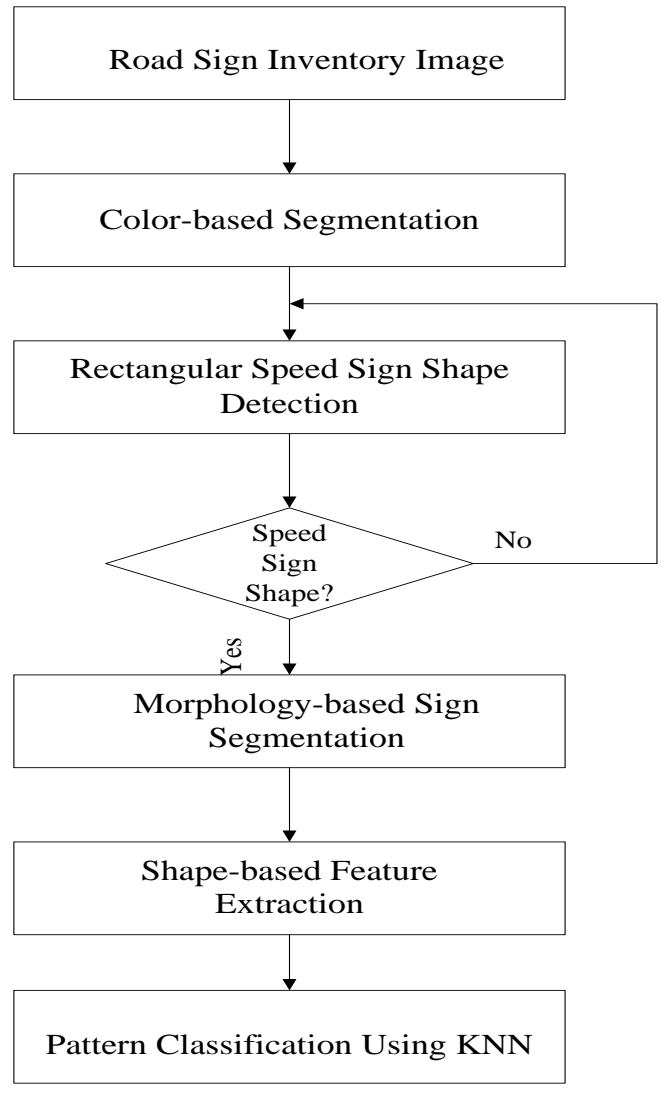

Figure 2: Flow diagram of the proposed system.

3. Morphology-based sign segmentation: this stage discards all objects (blobs) from the detected binary image except the numerals by applying a series of morphological operations.

4. Shape-based feature extraction: shape descriptors are used to build a feature vector of the speed sign numeral objects.

5. Pattern classification using $\mathrm{KNN}$ : this stage classifies speed signs to their categories using KNN classifier with the shape feature vector of numerals. Classification accuracy is estimated using leave-one-out (LOO) crossvalidation procedure.

\subsection{Color-based Segmentation}

Road sign inventory image would be segmented to obtain a binary image by extracting white color objects. The objects of this binary image would be tested in the next stage to detect rectangular speed sign objects.

This color segmentation has been achieved using the chromatic/achromatic decomposition technique suggested in [11] to generate a gray image (achromatic image) with no color information. Color thresholding is then applied on the gray image (Achromatic image) to segment the bright objects which are most likely belongs to the white speed limit sign.

\subsection{Rectangular Speed Sign Shape Detection}

This stage accepts or discards speed sign objects forwarded from the color segmentation by applying a set of geometric detectors suggested in [12]. Four cascaded geometric detectors are used to check each object in the binary image forwarded from the previous stage:
1. Area: this detector is used to discard all small and large objects from the segmented frame according to specific thresholds.

2. Solidity: this is defined as the ratio between number of ROI background pixels and the total number of ROI pixels. This detector is used to discard extremely solid and extremely non-solid objects.

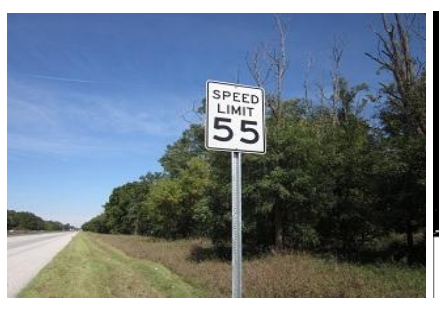

(a)

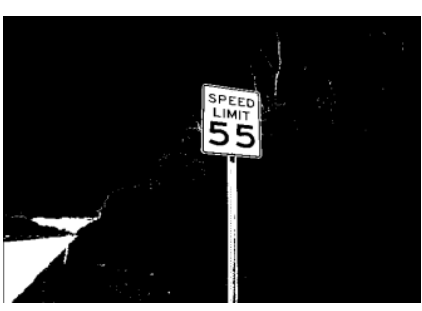

(b)

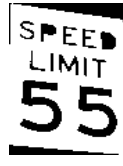

(c)

Figure 3: Shape detection process of speed sign. a) original frame. b) segmented frame. c) detected speed sign.

3. Vertices relative positions: this detector compares the relative positions of object vertices and has the ability to detect rotated and non-rotated objects.

4. Dimensions ratio: each object pass the previous three geometric constraints should satisfy preselected thresholds of dimensions ratio to discard non-symmetric objects and maintain rectangular shape objects.

The output of this stage is a rectangular shape object that satisfies the geometric detectors constraints of speed limit signs as shown in Figure 3.

\subsection{Morphology-based Sign Segmentation}

The main purpose of this stage is to remove irrelevant and nonedistinguishing objects that may be present in the detected sign More specifically, the segmentation step is designed to restore the central portion (two digits or numerals), which are the most discriminative patterns of the input sign image.

A series of binary and morphological image operations are applied to discard all speed sign objects rather than numerals. These operations include image inversion (or negative), sign border removal using a binary mask, and successive image erosion and dilation operation to improve image segmentation. Lastly, a morphological opening using a small structuring element is used to break undesired narrow region connectivity. Image segmentation is usually challenging with many false object produced. To further improve the segmentation and to eliminate false and unwanted objects, shape or blob analysis is employed. This process is initiated by labeling the connected regions in the segmented image; then, for each labeled region, shape descriptors such as area or size, centroid, eccentricity, and boundary extrema points are computed. It is worth noticing that these shape measurements will be used by the feature extraction stage. Figure 4 shows the steps followed in this stage in addition to the next two stages. 
For U.S. speed signs in particular, as shown in Figure 5, the most discriminative patterns are the two digits (numerals). Such patterns are relatively larger in size and have more importance than other patterns in the sign image. This fact is used to reduce the computational complexity and to improve the recognition process by ranking segmented blobs according to their size and by selecting the two blobs (or regions) with the largest size. All candidate blobs (regions) are further examined using location (boundary exterma and centroid) and area shape descriptors to reduce the number of blobs to two by rejecting the region with weak candidacy. The shape features of these two blobs (which represent the numeral

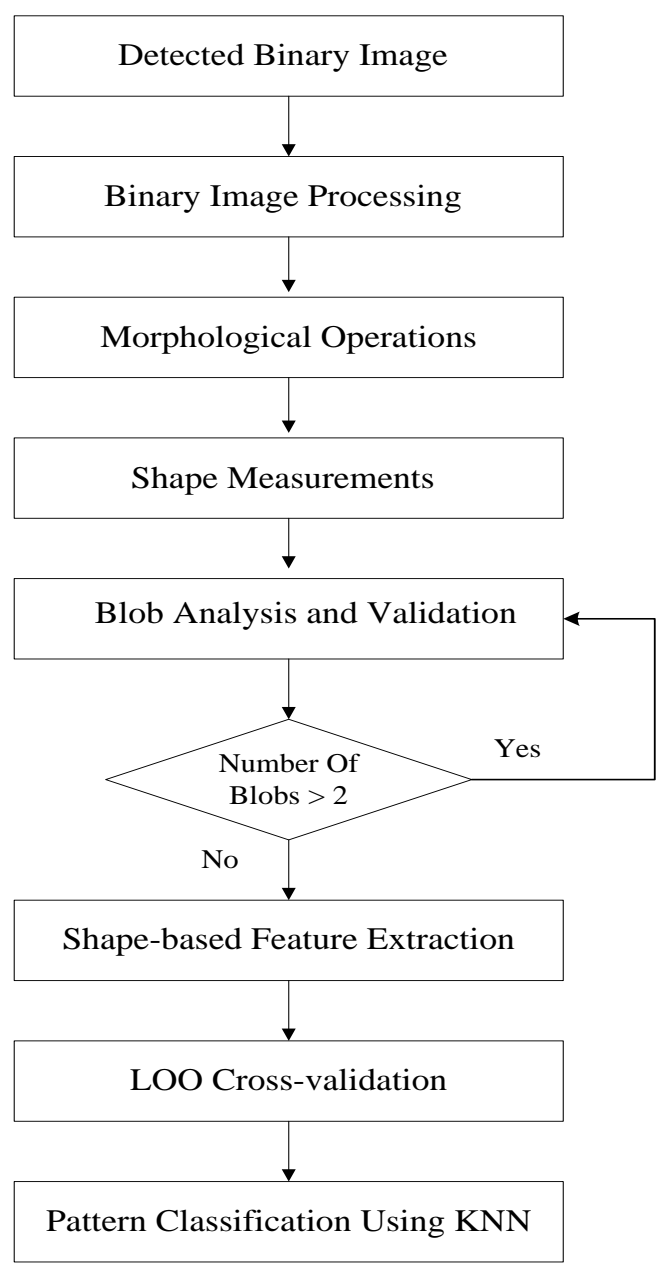

Figure 4: Shape-based speed sign recognition.

objects) would be extracted in the next stage since they have enough features to differentiate rectangular speed signs.

\subsection{Shape-based Feature Extraction}

From each blob a set of 10 features are directly or indirectly measured. This produces a total of 20 shape features representing each road sign since each segmented speed sign contains two blobs. The complete set of shape descriptors used for feature extraction is listed in Table 1. In addition to standard shape descriptors, three region descriptors are also computed and used:

- Compactness, this regional descriptor measures the circularity of the region and is defined as

$$
\begin{aligned}
& \text { Compactness } \\
& =\frac{4 \pi \text { Area }^{2}}{\text { Perimeter }^{2}}
\end{aligned}
$$

- $\quad$ Normalized deviation of radial distances (NDRD), this new shape descriptor measures how the eight exterma points of the region boundary are distributed, which is computed as follows

$$
\begin{aligned}
& R D=\sqrt{\left(x_{i}-C_{x}\right)^{2}+\left(y_{i}-C_{y}\right)^{2}} \\
& i=1,2, \ldots, 8 \\
& N D R D=\quad \frac{\operatorname{Std}(R D)}{\text { Area }}
\end{aligned}
$$

where $C_{x}, C_{y}$ are the spatial coordinates of the region centroid, $x_{i}, y_{i}$ with $i=1,2, \ldots 8$ are the eight extrema points located along the region boundary and $S t d(R D)$ is the standard deviation of. To make this descriptor invariant to scale, the region area is used for normalizing the deviation of the radial distances (RDs).

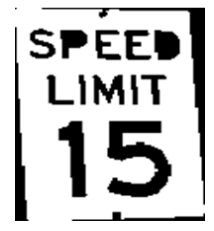

(a)

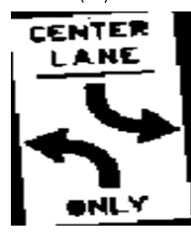

(e)

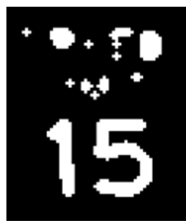

( b)

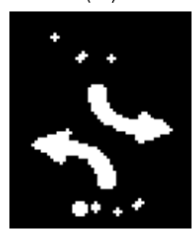

(f)

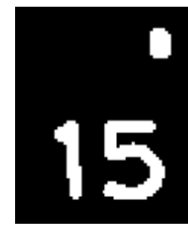

(c)

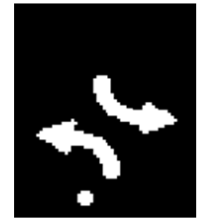

( g)

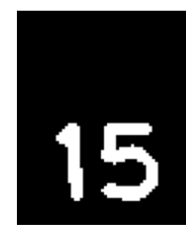

(d)

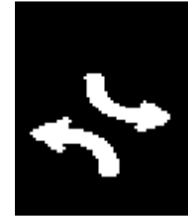

( f)
Figure 5: Shape based pattern segmentation of U.S. Road Signs: Input images are in (a) and (e), the results of binary and morphological operations are in (b) and (f), the outcomes of the area-based blob ranking and elimination are demonstrated in (c) and (f), and the segmented sign numerals (discriminating patterns )are shown in (d) and (f).

- Region major and minor axial difference: this measure is computed as the difference between the region major and minor axises.

\subsection{Pattern Classification using KNN}

In this work, a nonparametric and simple supervised learning based k-nearest neighbor classifier (KNN) is used for the discrimination of different speed sign patterns. The extracted shape features are used as input to the KNN classifier. The correct classification rate or classification accuracy is estimated using leave-one-out (LOO) cross-validation procedure as a metric for evaluating the performance of the proposed recognition system.

Given a dataset of $N$ samples, using the LOO cross-validation procedure, one sample is used for testing while the remaining $\mathrm{N}-1$ samples are used for training. This training-testing process is repeated until all samples are used for testing. In addition to the fact that LOO procedure provides an unbiased estimate of the generalization capacity of supervised leaning methods, the 
small number of samples, used in this work, is another reason for using a LOO cross-validation procedure rather than portioning the data into training and testing sets.

\section{EXPERIMENTAL RESULTS 3.1 Speed sign data set}

The proposed speed sign recognition is tested using 195 rectangular speed sign images categorized as: 27 signs of speed limit 15, 33 signs of speed limit 25, 22 signs of speed limit 35, 31 signs of speed limit 45, 39 signs of speed limit 55, and 44 signs of other sign images, which are also rectangular and of white color road signs but showing traffic sign other than previous speed limit signs. These road signs have been detected in traffic scenes captured using SAMSUNG ST65 camera in addition to images from VISAT ${ }^{\mathrm{TM}}$ Mobile Mapping System. Moreover, the proposed speed limit recognition system including the image segmentation, shape based feature extraction, and pattern classification using $\mathrm{KNN}$ is implemented in MATLAB software running on 2.4-GHz i3 CPU.

Table 1. List of Shape Descriptors used for Speed Sign Recognition.

\begin{tabular}{|c|c|}
\hline $\begin{array}{l}\text { Shape } \\
\text { Measure }\end{array}$ & Descriptions \\
\hline Area & $\begin{array}{l}\text { Estimated by counting the number of the } \\
\text { pixels in the region. }\end{array}$ \\
\hline Eccentricity & $\begin{array}{l}\text { A measure of the region longitude. } \\
\text { Theoretically, its value ranges from } 0 \text { for a } \\
\text { circular region to } 1 \text { in case of linear region. }\end{array}$ \\
\hline Extent & $\begin{array}{l}\text { Measured as the ratio of the region area to the } \\
\text { bounding box area. }\end{array}$ \\
\hline Solidity & $\begin{array}{l}\text { Measured as the ratio of region area to convex } \\
\text { area. }\end{array}$ \\
\hline $\begin{array}{l}\text { Minor } \\
\text { /Major Axis } \\
\text { length }\end{array}$ & $\begin{array}{l}\text { Measured as the length (in pixels) of the } \\
\text { minor/major axis of the ellipse that has the } \\
\text { same normalized second central moments as } \\
\text { the region. }\end{array}$ \\
\hline $\begin{array}{c}\text { Major and } \\
\text { minor axial } \\
\text { difference }\end{array}$ & $\begin{array}{l}\text { Computed by subtracting the region major } \\
\text { axis from the minor axis. }\end{array}$ \\
\hline Compactness & $\begin{array}{l}\text { Measured as the ratio of squared area of the } \\
\text { region to its perimeter (region circularity). }\end{array}$ \\
\hline Convex Area & $\begin{array}{l}\text { Measures the number of pixels in smallest } \\
\text { convex polygon region that can fit in the } \\
\text { region. }\end{array}$ \\
\hline $\begin{array}{l}\text { Normalized } \\
\text { Deviation of } \\
\text { Radial } \\
\text { Distances }\end{array}$ & $\begin{array}{l}\text { This measures how the eight exterma points of } \\
\text { the region are radially distributed along the } \\
\text { region boundary. }\end{array}$ \\
\hline
\end{tabular}

\subsection{Results Analysis}

The results of applying the proposed shape based speed sign recognition are presented in Table 2. Results indicated excellent performance of the proposed shape based recognition system. The system also continues to provide an acceptable average classification performance over wide range of classifier parameters.
Table 2. Average classification accuracy of the proposed speed sign recognition system.

\begin{tabular}{|c|l|}
\hline $\begin{array}{c}\text { KNN } \\
\text { Parameter }\end{array}$ & $\begin{array}{c}\text { Classification } \\
\text { Accuracy }\end{array}$ \\
\hline 1 & $0.87 \pm 0.03$ \\
\hline 3 & $0.88 \pm 0.02$ \\
\hline 5 & $0.88 \pm 0.02$ \\
\hline 7 & $0.88 \pm 0.02$ \\
\hline 9 & $0.87 \pm 0.02$ \\
\hline 11 & $0.86 \pm 0.02$ \\
\hline 13 & $0.84 \pm 0.03$ \\
\hline 15 & $0.81 \pm 0.03$ \\
\hline
\end{tabular}

For an application like driver assistance system, the time needed for processing the input image frame and for the processing and recognition of existed road sign must support a real time deployment of the system. Although the current implementation of the proposed speed sign recognition system is not optimized for real-time setting, the computational complexity (evaluated in terms of execution time) of various stages of the system is promising. The average time required for executing each stage of the system is presented in Table 3 , which demonstrates that it takes the system about 66 milliseconds to accomplish the entire recognition process.

Table 3. Average Execution time for different stages of the proposed shape based speed sign recognition system.

\begin{tabular}{|l|l|}
\hline \multicolumn{1}{|c|}{$\begin{array}{c}\text { Sign Recognition } \\
\text { Stage }\end{array}$} & \multicolumn{1}{c|}{$\begin{array}{c}\text { Time } \\
\text { (milliseconds) }\end{array}$} \\
\hline Patten Segmentation & 31.33 \\
\hline Shape Feature Extraction & 32.81 \\
\hline KNN Classification & 1.94 \\
\hline Sign recognition of all stages & 66.08 \\
\hline
\end{tabular}

Feature selection, in general, is an important step toward better pattern recognition system, more efficient feature extraction, and also more efficient recognition system implementation and realization. Although the speed sign recognition presented in this work does not employ any feature selection technique, we have applied a simple backward feature elimination to get some insight of the significance of different shape descriptors used in this work. Using this backward feature elimination method, the classification performance is obtained using all features, then, one shape feature is removed at a time and the performance is evaluated again and compared.

The results of applying this process are presented in Table 4 . From these results, it can be noticed that eliminating a single feature, in most cases, has slightly affected the classification performance except the NDRD shape descriptor. For example, eliminating the eccentricity descriptor has degraded the average classification accuracy by about $1 \%$ while the removal of extent descriptor improves the performance by about $1 \%$. Further, the average performance is degraded by about $5 \%$ when the NDRD descriptor is eliminated. That is to say, the used shape features are jointly important and contributing to the obtained classification performance. 
* Region major and minor axial difference

Table 4. Impact of backward feature elimination on the classification performance.

\begin{tabular}{|l|c|c|c|}
\hline \multirow{2}{*}{ Shape Descriptor } & \multicolumn{3}{|c|}{ KNN Parameter } \\
\cline { 2 - 4 } & 1 & 5 & 11 \\
\hline Area & $0.87 \pm 0.02$ & $0.87 \pm 0.02$ & $0.85 \pm 0.02$ \\
\hline Extent & $0.88 \pm 0.02$ & $0.89 \pm 0.02$ & $0.85 \pm 0.02$ \\
\hline Solidity & $0.88 \pm 0.03$ & $0.87 \pm 0.02$ & $0.84 \pm 0.03$ \\
\hline Convex Area & $0.85 \pm 0.02$ & $0.87 \pm 0.03$ & $0.85 \pm 0.03$ \\
\hline Eccentricity & $0.87 \pm 0.02$ & $0.86 \pm 0.02$ & $0.85 \pm 0.02$ \\
\hline Circularity & $0.88 \pm 0.02$ & $0.87 \pm 0.03$ & $0.85 \pm 0.03$ \\
\hline Major Axis(MAJX) & $0.87 \pm 0.02$ & $0.87 \pm 0.02$ & $0.86 \pm 0.02$ \\
\hline Minor Axis(MINX) & $0.86 \pm 0.02$ & $0.89 \pm 0.02$ & $0.83 \pm 0.03$ \\
\hline MAJX-MINX* & $0.87 \pm 0.02$ & $0.87 \pm 0.02$ & $0.85 \pm 0.02$ \\
\hline NDRD & $0.83 \pm 0.03$ & $0.84 \pm 0.03$ & $0.84 \pm 0.03$ \\
\hline
\end{tabular}

\section{CONCLUSIONS}

A recognition system of speed signs used in the U.S has been proposed in this paper. In the detection phase, a set of cascaded detectors proposed in [12] for the detection of rectangular speed sign shapes has been deployed. Shape and morphological features of speed sign numerals have been extracted and used for the classification of speed signs.

The proposed system shows a high recognition rate in the experimental results in addition to low computational complexity of the recognition process. Moreover, obtained results show the robustness of the recognition performance to the variation of the classifier's parameter. The application of backward feature elimination has demonstrated the importance of NDRD shape descriptor compared to the other shape descriptors.

Further validation and improvement of the proposed system includes: 1) increasing the size of speed sign dataset; 2) improving the efficiency of sign segmentation by applying other morphology operations; 3) exploring new and more robust methods for feature extraction and classification; and 4) implementing and testing the system using real-time settings.

\section{REFERENCES}

[1] P.Gil-Jime'nez, S.Maldonado-Bascón, H.Go' mez-Moreno, S. Lafuente-Arroyo,F.Lo'pez-Ferreras, "Traffic sign shape classification and localization based on the normalized FFT of the signature of blobs and 2D homographies," Elsevier Signal Processing 88 (12), 2943-2955, 2008.

[2] S. Maldonado-Bascón, S. Lafuente-Arroyo, P. Gil-Jiménez, H. Gómez- Moreno, and F. López-Ferreras, "Road-sign detection and recognition based on support vector machines," IEEE Trans. Intell. Transp. Syst.,vol. 8, no. 2, pp. 264-278, Jun. 2007.

[3] A. de la Escalera, L.E. Moreno, M.A. Salichs, J.M. Armingol, "Road traffic sign detection and classification," Industrial Electronics, IEEE Transactions on , vol.44, no.6, pp.848-859, Dec 1997.
[4] I. Sebanja, D.B. Megherbi, "Automatic detection and recognition of traffic road signs for intelligent autonomous unmanned vehicles for urban surveillance and rescue," Technologies for Homeland Security (HST), 2010 IEEE International Conference on , vol., no., pp.132-138, 8-10 Nov. 2010.

[5] A. de la Escalera*, J.MaArmingol, M. Mata, "Traffic sign recognition and analysis for intelligent vehicles," Image and Vision Computing 21 247-258,2003.

[6] Yok-Yen Nguwi, A.Z. Kouzani, "Automatic Road Sign Recognition Using Neural Networks," Neural Networks, IJCNN '06. International Joint Conference on , vol., no., pp.3955-3962, 0-0 0,2006.

[7] Pedro Gil Jiménez, Saturnino Maldonado Bascón, HilarioGo'mezMoreno, SergioLafuente Arroyo, Francisco Lo' pezFerreras, "Traffic sign shape classification and localization based on the normalized FFT of the signature of blobs and 2D homographies," Signal Process.88, 12, 2943-2955,2008.

[8] J.F. Khan, S.M.A. Bhuiyan, R.R. Adhami, "Image Segmentation and Shape Analysis for Road-Sign Detection," Intelligent Transportation Systems, IEEE Transactions on , vol.12, no.1, pp.83-96, March 2011.

[9] X. Baro, S. Escalera, J. Vitria, O. Pujol and P. Radeva, "Traffic Sign Recognition Using Evolutionary Adaboost Detection and Forest-ECOC Classification," Intelligent Transportation Systems, IEEE Transactions on, vol. 10, pp. 113-126, 2009.

[10] Jialin Jiao, Zhong Zheng, Jungme Park, Y.L. Murphey, Yun Luo, "A robust multi-class traffic sign detection and classification system using asymmetric and symmetric features," Systems, Man and Cybernetics, 2009. SMC 2009. IEEE International Conference on , vol., no., pp.3421-3427, 11-14 Oct. 2009.

[11] H. Gomez-Moreno, S. Maldonado-Bascon, P. Gil-Jimenez, S. Lafuente-Arroyo, "Goal Evaluation of Segmentation Algorithms for Traffic Sign Recognition," Intelligent Transportation Systems, IEEE Transactions on , vol.11, no.4, pp.917-930, Dec. 2010.

[12] Abukhait, J.; Abdel-Qader, I.; Jun-Seok Oh; Abudayyeh, O., "Road sign detection and shape recognition invariant to sign defects," Electro/Information Technology (EIT), 2012 IEEE International Conference on , vol., no., pp.1,6, 6-8 May 2012.

[13] N. Barnes, A. Zelinsky and L. S. Fletcher, "Real-Time Speed Sign Detection Using the Radial Symmetry Detector," Intelligent Transportation Systems, IEEE Transactions on, vol. 9, pp. 322-332, 2008.

[14] Yok-Yen Nguwi and Siu-Yeung Cho, "Two-tier selforganizing visual model for road sign recognition," in Neural Networks, 2008. IJCNN 2008. (IEEE World Congress on Computational Intelligence). IEEE International Joint Conference, pp. 794-799,2008.

[15] P. Medici, C. Caraffi, E. Cardarelli, P. P. Porta and G. Ghisio, "Real time road signs classification," in Vehicular Electronics and Safety, 2008. ICVES 2008. IEEE International Conference, pp. 253-258,2008.

[16] S. Vitabile, A. Gentile and F. Sorbello, "A neural network based automatic road signs recognizer," in Neural 
Networks, 2002. IJCNN '02. Proceedings of the 2002 International Joint Conference , pp. 2315-2320,2002.

[17] M. A. Garcia-Garrido, M. A. Sotelo and E. MartmGorostiza, "Fast traffic sign detection and recognition under changing lighting conditions," in Intelligent Transportation Systems Conference, 2006. ITSC '06. IEEE, pp. 811-816,2006.

[18] J. Miura, T. Kanda and Y. Shirai, "An active vision system for real-time traffic sign recognition," in Intelligent Transportation Systems, 2000. Proceedings, IEEE, pp. 5257,2000 .

[19] E. Perez and B. Javidi, "Nonlinear distortion-tolerant filters for detection of road signs," Vehicular Technology, IEEE Transactions on, vol. 51, pp. 567-576, 2002.

[20] A. Soetedjo and K. Yamada, "Traffic Sign Classification Using Ring Partitioned Method," IEICE Trans.Fundam.Electron.Commun.Comput.Sci., vol. E88A, pp. 2419-2426, sep, 2005.

[21] Min Shi, Haifeng Wu and H. Fleyeh, "Support vector machines for traffic signs recognition," in Neural
Networks, 2008. IJCNN 2008. (IEEE World Congress on Computational Intelligence). IEEE International Joint Conference, pp. 3820-3827, 2008.

[22] T. Hassan and N. El-Sheimy, "Automated traffic sign detection for modern driver assistance systems," in FIG Congress, Sydney, Australia, 2010.

[23] A. Ruta, Y. Li and X. Liu, "Real-time traffic sign recognition from video by class-specific discriminative features," Pattern Recogn., vol. 43, pp. 416-430, jan, 2010.

[24] A. Ruta, Y. Li and X. Liu, "Traffic sign recognition using discriminative local features," in Proceedings of the 7th International Conference on Intelligent Data Analysis, Ljubljana, Slovenia, pp. 355-366, 2007.

[25] C. Bahlmann, Y. Zhu, Visvanathan Ramesh, M. Pellkofer and T. Koehler, "A system for traffic sign detection, tracking, and recognition using color, shape, and motion information," in Intelligent Vehicles Symposium, IEEE Proceedings, pp. 255-260,2005. 